\title{
Automatic Characterization of Myocardial Perfusion in Contrast Enhanced MRI
}

\author{
Vincenzo Positano \\ Institute of Clinical Physiology, National Research Council, Via Moruzzi, 1 Loc San Cataldo, 56100 Pisa, Italy \\ Email: positano@ifc.cnr.it \\ Maria Filomena Santarelli \\ Institute of Clinical Physiology, National Research Council, Via Moruzzi, 1 Loc San Cataldo, 56100 Pisa, Italy \\ Email:santarel@ifc.cnr.it \\ Luigi Landini \\ Department of Information Engineering, University of Pisa, Via Diotisalvi 2, 56122 Pisa, Italy \\ Email:llandini@ifc.cnr.it
}

Received 29 January 2002 and in revised form 16 October 2002

\begin{abstract}
The use of contrast medium in cardiac MRI allows joining the high-resolution anatomical information provided by standard magnetic resonance with functional information obtained by means of the perfusion of contrast agent in myocardial tissues. The current approach to perfusion MRI characterization is the qualitative one, based on visual inspection of images. Moving to quantitative analysis requires extraction of numerical indices of myocardium perfusion by analysis of time/intensity curves related to the area of interest. The main problem in quantitative image sequence analysis is the heart movement, mainly due to patient respiration. We propose an automatic procedure based on image registration, segmentation of the myocardium, and extraction and analysis of time/intensity curves. The procedure requires a minimal user interaction, is robust with respect to the user input, and allows effective characterization of myocardial perfusion. The algorithm was tested on cardiac MR images acquired from voluntaries and in clinical routine.
\end{abstract}

Keywords and phrases: magnetic resonance imaging, image registration, contrast-enhanced MRI, myocardial perfusion, mutual information.

\section{INTRODUCTION}

The use of contrast medium (CM) to enhance the information provided by magnetic resonance is a growing technique. In fact, the use of contrast-enhanced images allows joining the high-resolution anatomical information provided by standard MR with functional information obtained by means of the diffusion of CM in tissues. An important example of the use of perfusion analysis in MRI is myocardial perfusion imaging with Gadolinium as a contrast agent that allows assessing the extent and type of coronary artery disease (CAD). In particular, cardiac MR images are going to be used in many studies to assess myocardial perfusion at rest and during pharmacological stress, showing potential for producing perfusion images at higher resolution than nuclear medicine techniques as positron emission tomography (PET) or single photon emission computer tomography (SPECT). Moreover, the use of MR technique allows 2D or 3D imaging without orientation constraint and avoids patient exposition to radiation.
In order to follow the perfusion of the CM in myocardial tissues, several images of the heart are acquired during time, starting from the injection of CM. Usually, several slices in short axis view are acquired in order to cover the entire left ventricle. The quality of the myocardial perfusion can be visually assessed by qualitative evaluation of the signal intensity in myocardium after the CM injection. The main limitations of the visual analysis are the lack of quantitative perfusion indexes and the presence of artifact due to intensity inhomogeneities in magnetic field and in the signal reception using a dedicated coil.

In order to perform a quantitative analysis of the myocardial perfusion, the signal intensity changes in the acquired images must be evaluated. Therefore, time/intensity $(T / I)$ curves are extracted, measuring the intensity value of each image pixel in the myocardium during time. The indices extracted from the $T / I$ curves (i.e., the slope value) were demonstrated to be a useful diagnostic tool in the diagnosis of CAD $[1,2,3]$. Quantitative evaluation of the contrast agent presence during time implies the finding of the 
corresponding pixels in all temporal frames. Usually, the acquisition protocol is made to obtain spatial alignment of all frames. Therefore, each pixel in an image frame should correspond to the pixels in the other frames with the same geometrical coordinates. In this case, the pixel selection can be done on only one image in the temporal sequence (i.e., the one with the best contrast-to-noise ratio). This surely enhances both the reliability and the performance of the analysis. Unfortunately, obtaining spatially aligned images is a difficult task in cardiac image acquisition, in which images misalignment due to patient breathing and poor ECG synchronization is commonly observed. Therefore, a misalignment correction, also named image registration, is often needed in postprocessing phase. A lot of methods were proposed to address the general medical image registration problem $[4,5,6]$. In MRI cardiac perfusion analysis, the traditional approach uses manual registration of cardiac images, using anatomical markers defined by an expert operator along all images in the temporal sequence $[1,7]$. Taking in account that a single multiphase 3D study can consist of hundreds of images, this procedure seems to be time consuming and affected by intraobserver and interobserver variability. It also requires the presence of an expert cardiologist to effectively identify the markers. Other authors $[8,9]$ proposed to extract some geometrical features (i.e., left ventricle cavity) from each frame and to perform the image registration by registering the extracted geometrical features. Manual or semiautomatic segmentation of the left ventricle is a time-consuming task and is affected by intraobserver and interobserver variability. On the other hand, effective full-automatic segmentation algorithms are not yet available for CM-enhanced cardiac MRI. In fact, automatic image segmentation algorithms are very sensitive to signalto-noise ratio that is usually low in CM-enhanced MRI due to the need for very short acquisition times. Delzescaux et al. [10] proposed a method based on the manual delineation of myocardium, right and left ventricle on one frame in the sequence. Then an algorithm based on template matching performs the sequence registration. Bidaut and Vallée [11] proposed a method based on the minimization of intrinsic differences between each image and a reference image coupled to a two-dimensional (i.e., three parameters) rigid body correction.

We prefer to apply voxel-based methods that operate directly on the image gray values and are effective in our problem due to the high degree of similarity between involved images. Moreover, this kind of methods are image independent, so can be applied also to image from different anatomical regions [12] and can be easily extended to the registration of $3 \mathrm{D}$ data set. Finally, the registration procedure does not require the presence of an expert operator. Along voxel-based methods, we select mutual information (MI) as registration parameters because this was demonstrated effective in a large set of applications.

The goal of this study is to apply a new automatic analysis procedure starting from contrast-enhanced MR images of the heart in order to correct the images misalignment in the postprocessing phase, segment the left ventricle wall, and

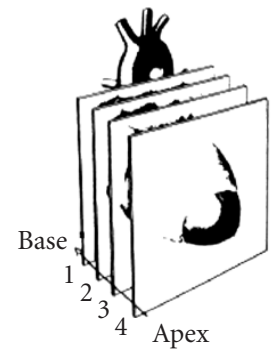

(a)

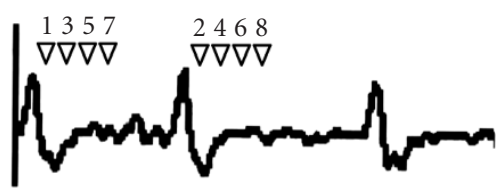

(b)
Figure 1: (a) Spatial and (b) temporal 3D data acquisition in FGRET sequence.

automatically extract the time/intensity curves and the related perfusion indexes.

\section{MATERIAL AND METHODS}

\subsection{MRI perfusion imaging protocol}

All exams are a set of Gd-DTPA contrast-enhanced cine cardiac MRI acquired by using a GE Signa Horizon LX system 1.5T. A cardiac array coil (4-elements-phased array) has been used as a radio frequency RF signal receiver, with cardiacgated fast gradient echo-echo train (FGRET) sequence. The patient under examination was asked to hold his breath during the examination. In order to perform a 3D acquisition, several parallel slices are acquired in short axis direction (Figure 1a). The image size is about $256 \times 256$ pixel with a planar resolution of about $1.2 \mathrm{~mm}$. In each individual heart beat (i.e., RR interval), a maximum of 5 slices can be acquired during the 300 millisecond interval positioned in diastole. In order to cover the entire left ventricle with a good spatial resolution along the short axis direction, $8-10$ slices are needed, so the $3 \mathrm{D}$ acquisition must be performed on two heart beats for each frame (Figure $1 \mathrm{~b}$ ). The $3 \mathrm{D}$ acquisition is repeated 30 to 40 times over time in order to follow the diffusion of the contrast agent.

Consequently, the time needed for a complete acquisition can reach $60-80$ second or more (60-80 RR intervals); in many cases the examination cannot be done in breathhold state. The acquired images are retrieved from the MR scanner via DICOM (digital imaging and communications in medicine) protocol.

\subsection{Automatic analysis}

The automatic analysis of the cardiac perfusion images obtained by CM-enhanced MRI can be summarized in three steps (Figure 2).

(1) The user selects the zone of interest on one frame. All the image sequence is automatically registered in order to correct the misalignments induced by patient breathing during examination.

(2) The left ventricle wall is segmented on one frame by a semiautomatic procedure. This procedure can be done 


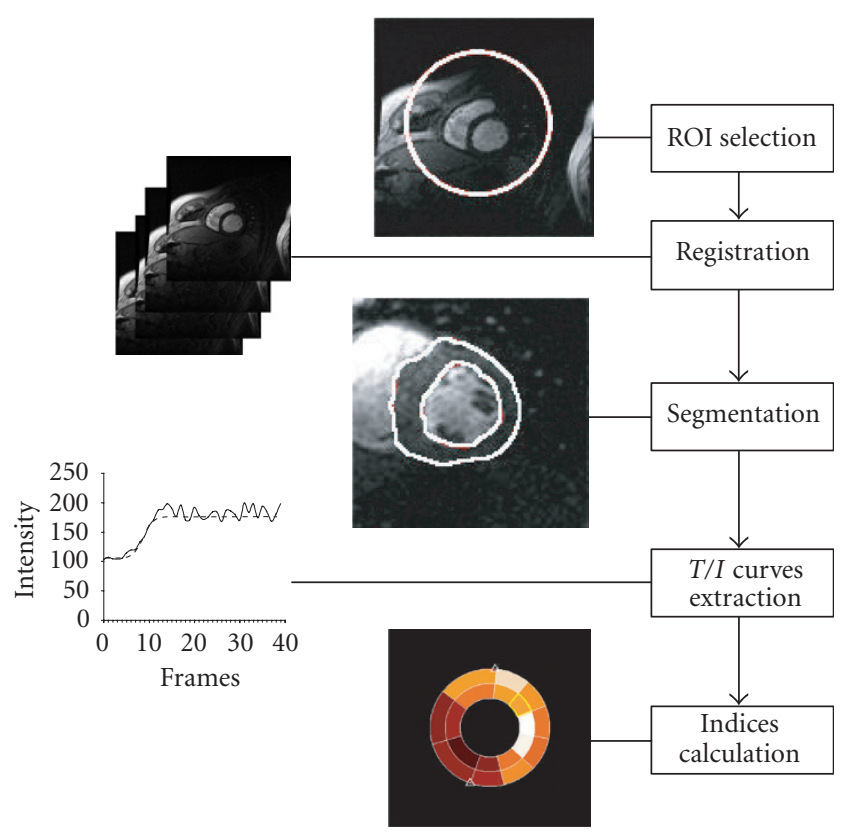

FIgURe 2: Automatic cardiac perfusion images analysis.

on only one frame, that is, the one with the best signalto-noise ratio.

(3) The segmented myocardium is automatically divided in regions of interest (ROIs). The user can select the number and the disposition of ROIs along the myocardium. For each ROI, the related time/intensity curves are extracted and perfusion indices are evaluated.

\subsection{Registration algorithm}

The MI concept comes from information theory, measuring the dependence between two variables or, in other words, the amount of information that one variable contains about the other [13]. In our application, we can identify the two variables with the gray levels distribution in two images to be aligned.

Let $Q$ and $K$ be images with $M$ pixels that can assume $N$ gray levels $g_{1}, g_{2}, \ldots, g_{N}$. The MI between $Q$ and $K$ can be defined as

$$
\operatorname{MI}(Q, K)=H(Q)+H(K)-H(Q, K),
$$

where $H(\cdot)$ is the entropy of an image. The entropy of $Q$ image $H(Q)$ can be written as

$$
H(Q)=-\sum_{i=1}^{N} \log \left[P\left(Q=g_{i}\right)\right] \cdot P\left(Q=g_{i}\right),
$$

$P\left(Q=g_{i}\right)$ means the probability that a pixel in $Q$ image will assume the value $g_{i}$ so that the image entropy can be written in terms of the image histogram $\operatorname{His}_{Q}$,

$$
H(Q)=-\frac{1}{M} \sum_{i=1}^{N} \log \left[\frac{\operatorname{His}_{Q}(i)}{M}\right] \cdot \operatorname{His}_{Q}(i) .
$$

As well, the joint entropy of two images $Q$ and $K$ with the same number of pixels $M$ and the same gray level range $N$ can be written in terms of joint image histogram $\mathrm{His}_{Q K}$,

$$
H(Q, K)=-\frac{1}{M^{2}} \sum_{i=1}^{N} \sum_{j=1}^{N} \log \left[\frac{\operatorname{His}_{Q K}(i, j)}{M^{2}}\right] \cdot \operatorname{His}_{Q K}(i, j) .
$$

The joint histogram $\operatorname{His}_{Q K}(i, j)$ is equal to the number of simultaneous occurrences of $Q=i$ and $K=j$.

The MI measures the relationship between two images. If the two images are independent, $H(Q, K)=H(Q)+H(K)$, and $\mathrm{MI}=0$. If one image provides some information about the second one, the MI becomes greater than zero.

The MI registration criterion states that the MI of the image intensity values of corresponding voxel pair is maximal if the images are geometrically aligned. Because no assumption is made about the nature of the relation between the image intensities, this criterion is very general and powerful $[14,15]$ so that it can be applied automatically at any image in the sequence also during CM transit.

In fact, in MR perfusion images the pixel values can change in dependence from the transit of CM. Instead, the statistics of gray levels distribution along the images remain almost the same, leading an MI-based registration effective with respect to other methods.

Let $Q$ be the reference image and $K$ the image that has to be registered with $Q$. The best rigid transformation $T$ that perform the registration can be found, maximizing the MI between $Q$ and $T(K)$, where $T(K)$ means the image obtained by the roto-translation of $K$ by the transformation matrix $T$,

$$
\operatorname{MI}[Q, T(K)]=H(Q)+H[T(K)]-H[Q, T(K)] .
$$

Finding the $T$ matrix that maximizes the value of MI implies the solution of an optimization problem with three (in case of 2D images) or six (in case of 3D images) variables.

In our problem, we have to find the best alignment not only between two images but also along all frames in the temporal sequence. In order to reduce the algorithm complexity and the related processing time, we choose to reduce the problem to a sequence of MI maximization between image/volume pairs. The way in which we choose the pairs will be shown in the following, now we can illustrate the registration algorithm between two images, as shown in Figure 3.

First, the MI between the reference image and the image under examination is evaluated. An optimization algorithm is used in order to estimate the best roto-translation matrix $(T)$; the matrix is used to rotate and translate the image. An interpolation operation is also required. If the result is satisfactory, the procedure ends; if not, a new roto-translation matrix is evaluated and a new loop is executed. A lot of work was done about medical image interpolation in order to find the best interpolation method [16]; we use two solutions: 


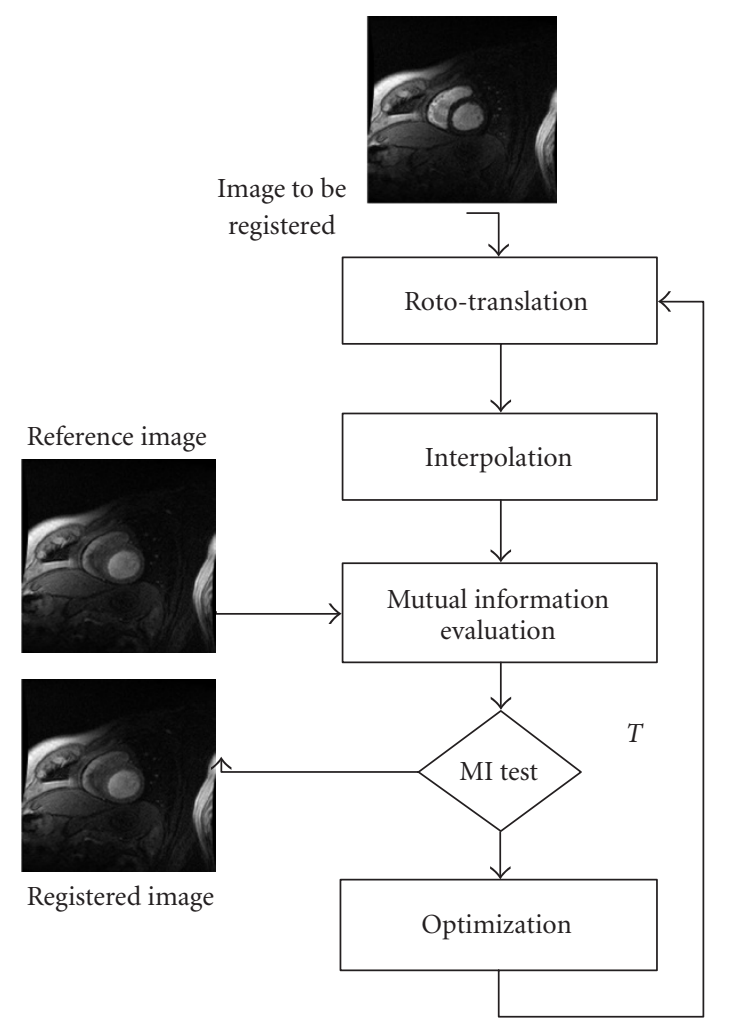

FIGURE 3: Flow chart of the registration algorithm.

a simple bilinear interpolation method during the MI maximum search to obtain the best time performance and an interpolation algorithm optimized for MR images [17] in the last step to compute the final volume.

The problem of finding the parameters set that maximizes a multivariable function is called optimization problem. The optimization algorithm should find the rotation and translation parameters that will maximize the MI. The main troubles are the presence of MI local maxima and the long processing time required by a lot of optimization algorithms.

We have tested two optimization algorithms, the simplex algorithm and the Powell algorithm [18], showing that the main advantage of the downhill simplex method is about time performance, because the simplex method requires only function evaluations — not derivatives — and so may be more reliable than other optimization methods. Instead, the Powell method is more effective with respect to the simplex method, especially for avoiding local maxima. On the other hand, it requires a long computation time.

In order to obtain an effective image registration, an important aspect is the way to choose the pair of images to be registered. The first idea is to register each image with the previous one. Because the CM diffuses in continuous manner, two consecutive images are almost similar in the sequence, so the registration algorithm can better correct the misalignment. On the other hand, an error in the registration of one image pair will affect the alignment of the whole temporal sequence. A second approach is to register all frames with respect to one image in the sequence. This one is selected by the user as the image that has to be used to perform the segmentation. Therefore, the user will select an image in which the ROI is well delineated. The user has also to roughly identify the left ventricle, surrounding it with a circular mask. Without the mask, the registration algorithm may try to register structures that do not belong to the heart region.

We have found that a registration with the second approach using the simplex method followed by a more accurate registration using the first approach and the Powell method leads in many cases to good results.

\subsection{Myocardium segmentation}

As shown in previous papers [19, 20, 21], anisotropic filtering of MR images joined with application of GVF-snake algorithm allows to effectively segment the endocardium and epicardium of the left ventricle.

The nonlinear anisotropic diffusion equation is

$$
\frac{\partial}{\partial t} I(\mathbf{x}, t)=\operatorname{div}[c(\mathbf{x}, t) \cdot \nabla I(\mathbf{x}, t)]
$$

The diffusion strength is controlled by $c(\mathbf{x}, t)$. The vector $\mathbf{x}$ represents the spatial coordinate, while the variable $t$ in our discrete implementation corresponds to iteration step $n$. The function $I(\mathbf{x}, t)$ is the image intensity. In order to preserve edges, the diffusion must be reduced or even blocked when close to a discontinuity.

We choose $c(\mathbf{x}, t)=g(|\nabla I(\mathbf{x}, t)|)$ to be a function of gradient magnitude evaluated on image intensity $I(\mathbf{x}, t)$,

$$
c(\mathbf{x}, t)=\frac{1}{2}[\tanh (\gamma(k-\|\nabla I(\mathbf{x}, t)\|))+1] .
$$

The parameter $\gamma$ controls the steepness of the min-max transition region, whereas $k$ controls the extent of the diffusion region in terms of gradient gray level. The parameter $\gamma$ can be fixed to 0.2 for 256 gray level images. In MR images processing, the $\gamma$ value has to be scaled proportionally to the range of the image values. In our application we use $\gamma=0.5$.

Starting from prefiltered images, a deformable model was developed as a curve that moves through the spatial domain of an image to minimize the following energy functional:

$$
E=\int_{0}^{1} \frac{1}{2}\left[\alpha\left|\mathbf{x}^{\prime}(s)\right|^{2}+\beta\left|\mathbf{x}^{\prime \prime}(s)\right|^{2}+E_{\text {ext }}(\mathbf{x}(s))\right] d s,
$$

where $\mathbf{x}(s)=[x(s), y(s)], s \in[0,1], \alpha$ and $\beta$ control the mechanical properties of the snake, that is, tension and rigidity, respectively, $\mathbf{x}^{\prime}$ and $\mathbf{x}^{\prime \prime}$ denote the first and the second derivatives of $\mathbf{x}(s)$ with respect to $s$, and $E_{\text {ext }}(\mathbf{x})$ is the potential associated to the external forces. External force $E_{\text {ext }}(\mathbf{x})$ is derived from the image gradient so that it takes on its smaller values at the edge points. The external force can be defined to be a vector field $\mathbf{v}(\mathbf{x})$ that minimizes the following functional:

$$
\varepsilon=\iint\left(\mu|\nabla \mathbf{v}|^{2}+|\nabla I|^{2}|\mathbf{v}-\nabla I|^{2}\right) d \mathbf{x}
$$


The optimal $\mu$ value is related with the signal-to-noise ratio and can be set to 0.2 in MRI imaging. This formulation forces the field to vary slowly in homogeneous regions and to keep $\mathbf{v}$ nearly equal to the gradient map where high spatial variations are present. In fact, the first term of (9) becomes dominant where $\nabla I(x, t)$ is small, yielding a slowing-varying field in homogeneous regions. On the other hand, the second term becomes dominant where $\nabla I(x, t)$ is large and is minimized by setting $\nabla v(x, t)=\nabla I(x, t)$. The external field $\mathbf{v}(\mathbf{x})$ resulting from this calculus of variations is used in $E$ expression as potential force $-\nabla E_{\text {ext }}(\mathbf{x})$, yielding [22]

$$
\mathbf{x}_{t}(s, t)=\alpha \mathbf{x}^{\prime \prime}(s)-\beta \mathbf{x}^{\prime \prime \prime \prime}(s)+\mathbf{v},
$$

where $\mathbf{x}^{\prime \prime}$ and $\mathbf{x}^{\prime \prime \prime \prime}$ are, respectively, the second and the fourth derivatives of $\mathbf{x}(s)$ with respect to $s$. We call the parametric deformable curve solving the previous equation the GVF snake. Values of $\alpha$ and $\beta$ parameters were tuned during the algorithm test on a large set of MRI images.

The semiautomatic segmentation of the myocardium can be done by the following steps.

(1) Anisotropic prefiltering of the MR image.

(2) Initialization of GVF snake procedure is made manually by tracing a very rough closed curve inside the $2 \mathrm{D}$ image at the level of ventricular cavity.

(3) The fitting of myocardium borders with a GVF snake is made as follows: (i) the GVF field is evaluated starting from an image edge map and an approximation to its gradient. Starting from the initial curve and fitting the first GVF field crest with a snake, the endocardial border is mathematically described; (ii) using the detected endocardial border as a new starting data set, the deformable model continues to search for a new curve that corresponds to a new local minimum of image energy. In particular, the GVF field experiences a second crest at this new local minimum which is used to describe the epicardial border with the snake model.

Figure 4 shows the results obtained after each algorithm phase. In particular, (a) is the starting image, (b) is the gradient map of the anisotropic filtered image, (c) is the map of the GVF module, and (d) is the original image with overimposing the two detected curves delimiting, respectively, the endocardial and epicardial borders.

The contours obtained by the previous procedure was copied along all frames. The users can manually modify the contours in order to correct errors introduced by the automatic registration procedure.

\subsection{Time/intensity curves extraction}

After the left ventricle wall segmentation, the user has to define a reference point (i.e., the anterior septal insertion of the right ventricle). The myocardium is automatically divided into the needed number of equiangular sectors starting from the reference point. Optionally, the myocardium can be divided into the inner and outer half (i.e., subendocardial and subepicardial layer). In this way, in each slice, the left ventricle wall can be automatically divided into a number of regions, ranging from 3 to 24, as shown in Figure 5.

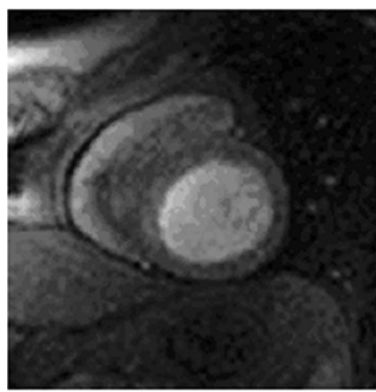

(a)

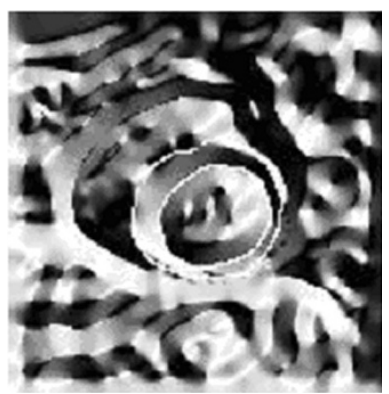

(c)

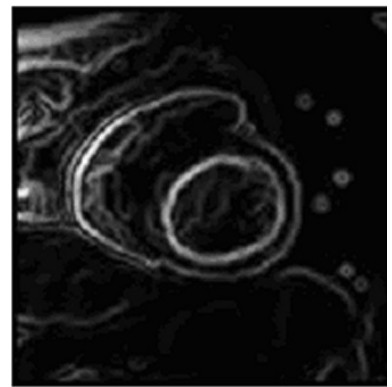

(b)

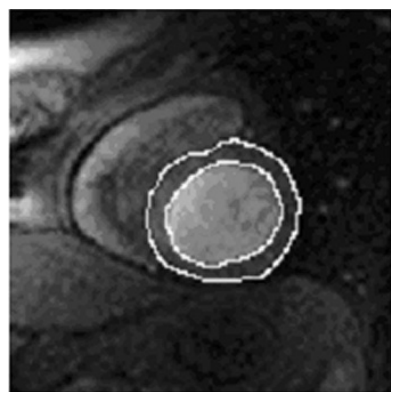

(d)
FIGURE 4: Segmentation algorithm phases: (a) starting image; (b) gradient of filtered image; (c) GVF image; and (d) segmented image.

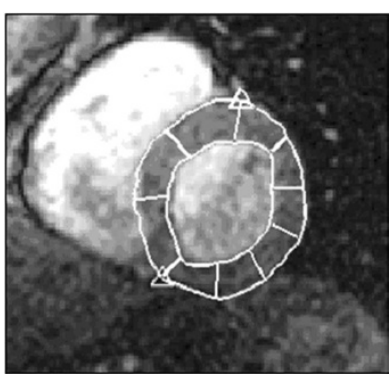

(a)

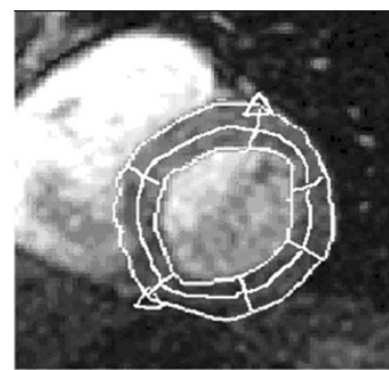

(b)
FIGURE 5: Automatic segmentation of the myocardial wall: (a) 9 sectors and (b) 6 sectors with subendocardial and subepicardial layers.

The $T / I$ curves are extracted for all pixels in all defined regions in segmented myocardium. For each region, the average $T / I$ curve is defined as the average along all $T / I$ curves in the region. Normalized $T / I$ curves are also evaluated by normalizing curves by the precontrast signal intensity.

Finally, average $T / I$ curve is automatically extracted from the center of the left ventricle cavity in order to evaluate the input function.

In order to extract quantitative indexes from $T / I$ curves, such curves have been fitted by using the ERF function 
TABLE 1: OA values evaluated before and after MI-based registration on voluntaries and patients.

\begin{tabular}{lcccc}
\hline & $\begin{array}{l}\text { Av. endo OA index without } \\
\text { registration }(\text { mean } \pm \text { SD) }\end{array}$ & $\begin{array}{l}\text { Av. endo OA index with } \\
\text { registration }(\text { mean } \pm \text { SD) }\end{array}$ & $\begin{array}{l}\text { Av. epi OA index without } \\
\text { registration }(\text { mean } \pm \text { SD) }\end{array}$ & $\begin{array}{l}\text { Av. epi OA index with } \\
\text { registration }(\mathrm{mean} \pm \mathrm{SD})\end{array}$ \\
\hline Voluntaries & $0.98 \pm 0.022$ & $0.98 \pm 0.025$ & $0.97 \pm 0.028$ & $0.97 \pm 0.031$ \\
Patients & $0.93 \pm 0.038$ & $0.97 \pm 0.015$ & $0.92 \pm 0.028$ & $0.97 \pm 0.013$ \\
\hline
\end{tabular}

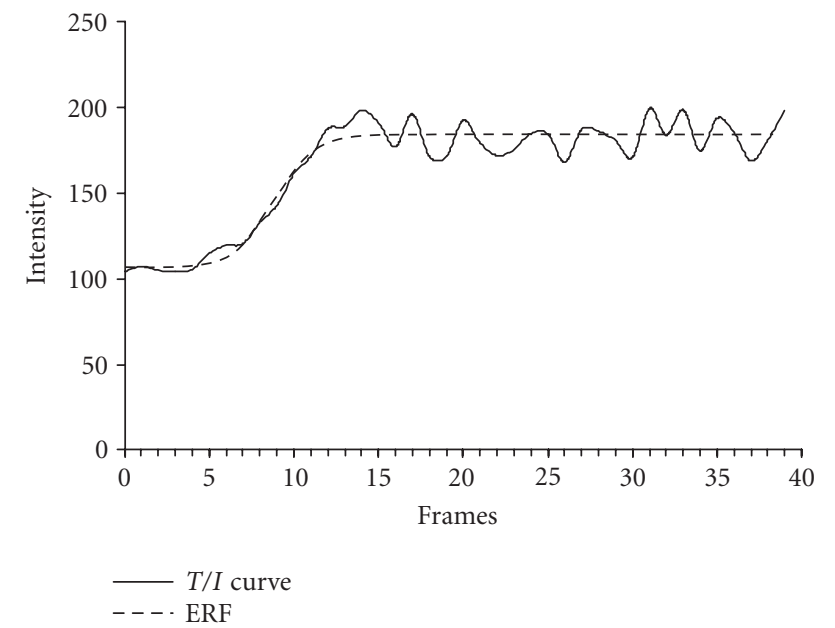

FIgURE 6: Example of a typical T/I average intensity curve and the related ERF function.

defined as

$$
\operatorname{erf}(x)=a_{2}+a_{3} \frac{2}{\sqrt{\pi}} \int_{0}^{x} e^{-\left(t-a_{1}\right)^{2} / a_{4}} d t
$$

The four parameters used in curve fitting are the ERF translation on $x$ and $y$ axes $\left(a_{1}\right.$ and $\left.a_{2}\right)$, the scaling $a_{3}$, and the slope $a_{4}$.

Figure 6 shows a typical T/I average intensity curve and the related ERF function.

Fitting with Gamma function is also available to examine images obtained by an intravascular CM, according to [23]. Useful perfusion indexes are then extracted and evaluated. They are wash-in slope, time to peak intensity, and peak value. These parameters are measured as absolute values and as relative values with respect to the input function for both original and normalized T/I curves. Manual correction of the ERF function is available: the user can manually cut off curve points in order to obtain a correct shape for the ERF curve.

\section{RESULTS}

The algorithm was implemented on interactive data language (IDL) release 5.4. IDL is widely used in medical community for data analysis, visualization, and crossplatform application development. The software implementing the proposed algorithm is available on request (consult http://nmr-aurora.ifc.cnr.it/imaging/hippo.html). The images in DICOM format produced by the MR device was transferred by a high-speed network to a workstation and analyzed by a cardiologist.

The method has been tested on two kinds of image data set. The first set was acquired from collaborative voluntaries, able to hold their breath and to reduce movements during the entire examination. The second data set was acquired from patients with suspected CAD scheduled for MRI examination. For each exam, a total of 245 images was acquired, consisting of 7 short axis slices, each one with 35 temporal frames acquired in diastolic phase. A total of 5 examinations on voluntaries and 5 examinations on patients were used for algorithm effectiveness evaluation. Therefore, a total number of 70 temporal image sequences was used.

In order to assess the effectiveness of the automatic registration procedure, an expert user was asked to use the program with and without the use of the automatic registration algorithm. For each spatial slice, the endocardial and epicardial contours have been obtained by the segmentation procedure previously described. The contours were replicated along all frames and the user was asked to manually correct the endocardial and epicardial borders. We used the overlapping area $(\mathrm{OA})$ index as the index of the needed correction degree. Overlapping area is the common area between the region selected in the developing image and the reference one, normalized by the reference area.

Table 1 shows the average values related to both voluntaries and patients.

Figure 7 shows the average value of $\mathrm{OA}$ index for each frame with and without registration on patient images. The value of OA index on patient images is reduced by the registration procedure and becomes comparable with the index measured on volunteer images.

Figure 8 shows an example of a T/I curve extracted from a myocardium region before and after application of the registration algorithm. The artefacts present in the T/I curve before registration are greatly reduced with the application of the MI-based registration algorithm. In particular, the algorithm was able to correct both the artefact produced by the pass of the CM in the right ventricle (frames 2-6) and the artefact produced by background signal (frames 2135).

Algorithm robustness with respect to the algorithm input was tested by the following procedure: five different operators were asked to perform the registration procedure on the same data set ( 7 slices, 35 frames). Each operator has to select the reference slice and to draw the ROI at the procedure beginning. For each test, the required computation time and the obtained roto-translation matrices for all frames were recorded. The mean processing time was 90.83 second 
TABLE 2: Algorithm robustness with respect to the user input.

\begin{tabular}{cccc}
\hline Operator & $\begin{array}{c}X \text { component }(\mathrm{mm}) \\
(\text { mean } \pm \text { SME) }\end{array}$ & $\begin{array}{c}Y \text { component }(\mathrm{mm}) \\
(\text { mean } \pm \text { SME) }\end{array}$ & $\begin{array}{c}\theta \text { component }(\mathrm{mm}) \\
(\text { mean } \pm \text { SME) }\end{array}$ \\
\hline 1 & $2.68 \pm 0.047$ & $4.42 \pm 0.35$ & $1.20 \pm 0.096$ \\
2 & $2.68 \pm 0.040$ & $4.42 \pm 0.20$ & $1.20 \pm 0.062$ \\
3 & $2.68 \pm 0.024$ & $4.42 \pm 0.25$ & $1.20 \pm 0.122$ \\
4 & $2.68 \pm 0.033$ & $4.42 \pm 0.15$ & $1.20 \pm 0.087$ \\
5 & $2.68 \pm 0.031$ & $4.42 \pm 0.18$ & $1.20 \pm 0.066$ \\
\hline
\end{tabular}

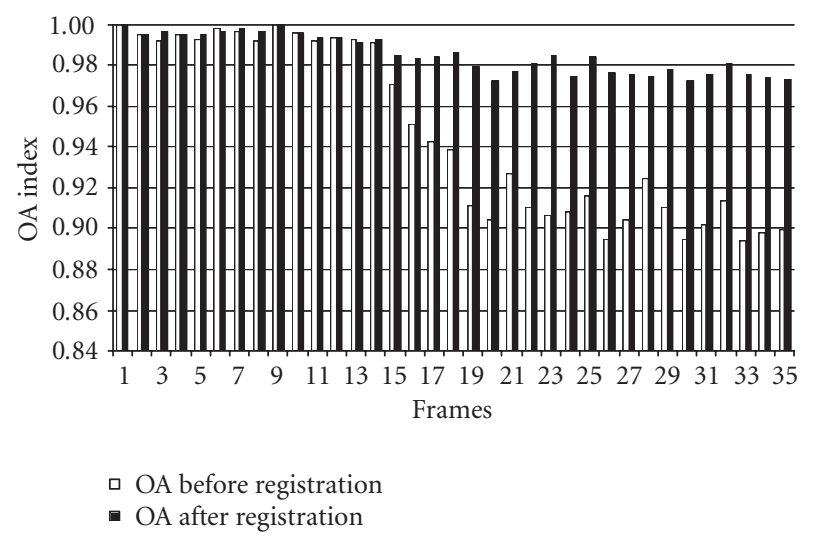

FIgURE 7: OA index before and after registration.

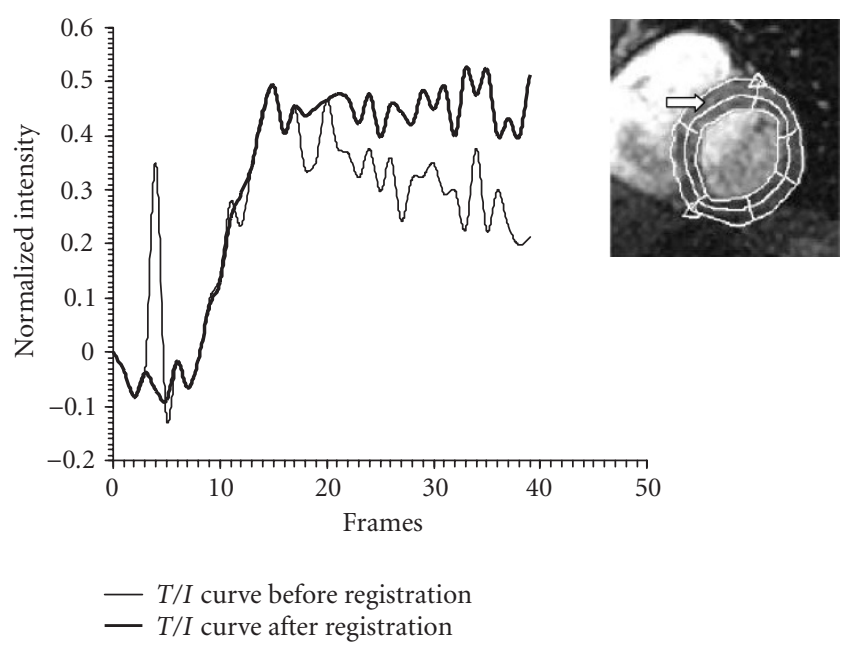

Figure 8: T/I curve extracted from a myocardium region before and after application of the registration algorithm.

with standard deviation of 3.60. For each component of the roto-translation matrix, the squared mean error with respect to the mean value was then evaluated. Table 2 shows the test result. Mean square error is measured in millimeters for $X$ and $Y$ components and degrees for $\theta$ component.

The algorithm seems to be robust with respect to the ini- tialization of the registration procedure, both in the final registration result and in the required processing times.

\section{DISCUSSION}

An algorithm for fast quantitative analysis of cardiac MR perfusion images was presented. The algorithm requires minimal user interaction and is robust with respect to the user input. The use of an automatic registration procedure based on maximization of the MI was demonstrated to be effective in order to address the requirement of fast and automatic tools for quantitative analysis of CM-enhanced MR images. The quantitative index OA was introduced in order to measure in a quantitative way the algorithm effectiveness. Results on cardiac images show that misalignments and artefacts introduced by patient movement during the examination are greatly reduced.

In this paper, our approach was to reduce the problem of contemporary registration of several temporal frames to a lot of registration operations between image pairs. The development of a global registration algorithm should improve the registration quality but the increasing of algorithm complexity can leads to unacceptable processing time.

The main limitation of our approach is the 2D nature of the image registration that does not allow correcting the misalignment component along the normal to the acquisition plane (i.e., $z$-axis). The proposed algorithm can be extended in $3 \mathrm{D}$ without main modification, but some problems should be solved. The first one is the low image resolution along the $z$-axis that implies the production of interpolation artefacts. The second one is the increasing in the algorithm complexity, because the optimization algorithm has to work on 6 instead of 3 parameters. Finally, slices related to a 3D volume are acquired in different times, as previously shown, so they are not homogeneous with respect to the intensity of the CM. In our opinion, the use of 3D analysis of myocardial perfusion MRI in clinical environment requires both an improvement in MR device technology (i.e., better resolution in $z$-axis direction) and in computer power (i.e., reduction of processing time).

\section{ACKNOWLEDGMENT}

The authors would like to thank Dr. Massimo Lombardi of the CNR Institute of Clinical Physiology in Pisa for collecting the cardiac perfusion MR images. 


\section{REFERENCES}

[1] J. Schwitter, D. Nanz, S. Kneifel, et al., "Assessment of myocardial perfusion in coronary artery disease by magnetic resonance: a comparison with positron emission tomography and coronary angiography," Circulation, vol. 103, no. 18, pp. 2230-2235, 2001.

[2] N. Wilke and M. Jerosch-Herold, "Assessing myocardial perfusion in coronary artery disease with magnetic resonance first-pass imaging," Cardiology Clinics, vol. 16, no. 2, pp. 227246, 1998.

[3] T. Laddis, W. J. Manning, and P. G. Danias, "Cardiac MRI for assessment of myocardial perfusion: current status and future perspectives," Journal of Nuclear Cardiology, vol. 8, no. 2, pp. 207-214, 2001.

[4] P. A. van den Elsen, E. J. D. Pol, and M. A. Viergever, "Medical image matching - a review with classification," IEEE Engineering in Medicine and Biology, vol. 12, no. 1, pp. 26-39, 1993.

[5] J. B. Maintz and M. A. Viergever, "A survey of medical image registration," Medical Image Analysis, vol. 2, no. 1, pp. 1-36, 1998.

[6] C. R. Maurer and J. M. Fitzpatrick, "A review of medical image registration," in Interactive Image Guided Neurosurgery, pp. 17-44, American Association of Neurological Surgeons, Park Ridge, Ill, USA, 1993.

[7] J. P. Vallee, H. D. Sostman, J. R. MacFall, et al., "MRI quantitative myocardial perfusion with compartmental analysis: a rest and stress study," Magnetic Resonance in Medicine, vol. 38, no. 6, pp. 981-989, 1997.

[8] X. Yang, Y. Taeymans, P. Carlier, P. Verstraeten, K. Brunfaut, and M. Cornelis, "Computer aided measurement of local myocardial perfusion in MRI," in Proc. Computers in Cardiology, pp. 365-368, 1993.

[9] G. Gerig, R. Kikinis, W. Kuoni, G. K. von Schulthess, and Kubler O., "Semiautomated ROI analysis in dynamic MR studies. Part I: Image analysis tools for automatic correction of organ displacements," Journal of Computer Assisted Tomography, vol. 15, no. 5, pp. 725-732, 1991.

[10] T. Delzescaux, F. Frouin, A. De Cesare, et al., "Adaptive and self-evaluating registration method for myocardial perfusion assessment," Magma, vol. 13, no. 1, pp. 28-39, 2001.

[11] L. M. Bidaut and J. P. Vallée, "Automated registration of dynamic MR images for the quantification of myocardial perfusion," J Magn Reson Imaging, vol. 13, no. 4, pp. 648-655, 2001.

[12] V. Positano, M. F. Santarelli, L. Landini, and A. Benassi, "Automatic time sequence aligment in contrast enhanced MRI by maximization of mutual information," in Proc. 23rd Annual International Conference of the IEEE Engineering in Medicine and Biology Society, pp. 1-4, Istanbul, Turkey, 2001.

[13] A. Papoulis, Probability, Random Variables, and Stochastic Processes, McGraw-Hill, New York, NY, USA, 3rd edition, 1991.

[14] P. Viola and W. M. Wells III, "Alignment by maximization of mutual information," International Journal of Computer Vision, vol. 24, no. 2, pp. 137-154, 1997.

[15] F. Maes, A. Collignon, D. Vandermeulen, G. Marchal, and P. Suetens, "Multimodality image registration by maximization of mutual information," IEEE Trans. on Medical Imaging, vol. 16, no. 2, pp. 187-198, 1997.

[16] G. J. Grevera and J. K. Udupa, "An objective comparison of 3D image interpolation methods," IEEE Trans. on Medical Imaging, vol. 17, no. 4, pp. 642-652, 1998.

[17] J. V. Hajnal, N. Saeed, E. J. Soar, A. Oatridge, I. R. Young, and G. M. Bydder, "A registration and interpolation procedure for subvoxel matching of serially acquired MR images," Jou. Comp. Ass. Tom., vol. 19, no. 2, pp. 289-296, 1995.

[18] V. Positano, M. F. Santarelli, L. Landini, and A. Benassi, "Automatic time sequence alignment in contrast enhanced MRI by maximization of mutual information," in Proc. 23rd Annual International Conference of the IEEE Engineering in Medicine and Biology Society, Istanbul, Turkey, October 2001.

[19] M. F. Santarelli, V. Positano, L. Landini, and A. Benassi, "A new algorithm for 3D-automatic detection and tracking of cardiac wall motion," in Computers in Cardiology, pp. 133136, IEEE Comp. Soc. Press, Los Alamitos, Calif, USA, 1999.

[20] M. F. Santarelli, V. Positano, L. Landini, et al., "A new method for automatic tracking and analysis of MR images of the heart," in Proc. International Society for Magnetic Resonance in Medicine, p. 1549, Denver, Colo, USA, 2000.

[21] V. Positano, M. F. Santarelli, L. Landini, and A. Benassi, "Nonlinear anisotropic filtering as a tool for SNR enhancement in cardiovascular MRI," in Computers in Cardiology, pp. 707710, IEEE, Cambridge, Mass, USA, 2000.

[22] C. Xu and J. L. Prince, "Snakes, shapes, and gradient vector flow," IEEE Trans. Image Processing, vol. 7, no. 3, pp. 359-369, 1998.

[23] W. H. Berninger, L. Axel, D. Norman, S. Napel, and R. W. Redington, "Functional imaging of the brain using computed tomography," Radiology, vol. 138, pp. 711-716, 1981.

Vincenzo Positano was born in Lecce, Italy, on May 4, 1964. He works as Researcher at the CNR Institute of Clinical Physiology (IFC-CNR) in Pisa, Italy. He received the Master degree in electronic engineering from the University of Pisa, Italy, in 1992. From 1993 to 1995, he worked as a Contract Researcher at the Department of Information Engineering of the Pisa University on parallel computing applied to electromagnetic scattering problems. Since 1995, he worked at the CNR Institute of Clinical Physiology in Pisa on parallel computing applications in medical imaging and automatic segmentation, registration, and quantitative analysis of MR image data. Currently, he is involved in developing innovative algorithms for images analysis in cardiac MR and fMRI fields.

Maria Filomena Santarelli was born in Rieti, Italy, on November 3, 1962. She received the Master degree in information science in 1987 from the University of Pisa. She has been a Research Fellow of Foundation for Biomedical Research at the CNR Institute of Clinical Physiology of Pisa from 1987 to 1989 . She received the Ph.D. degree in biomedical engineering in 1993 from Engineering Faculty of Pisa University. From

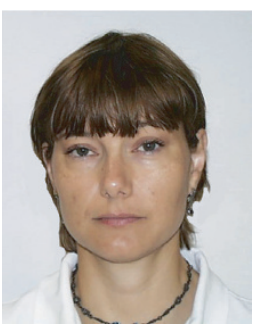
1994 to 1996, she was a Postdoctoral Fellow at the Department of Information Engineering of Pisa University. Since 2000, she teaches regular courses on medical informatics and programming at Biomedical Engineering Degree of Pisa University. She is currently a Biomedical Engineering Researcher at the CNR Institute of Clinical Physiology of Pisa. Her research activity is mainly on biomedical signal and image processing. She has published a number of proceedings papers and papers on medical image processing and tissue characterization. 
Luigi Landini was born in La Spezia, Italy, on October 31, 1949. He received the Master degree in physics from Pisa University, Italy, in 1974. He was Research Fellow at the CNR Institute of Clinical Physiology of Pisa from 1975 to 1979 , and at the Centro "E. Piaggio" of the Engineering Faculty of Pisa University from 1979 to 1981 . In 1981, he joined the Assistant Professor position at the Engineering Faculty of Pisa. He is actually an

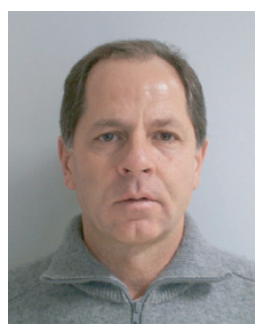
Associate Professor in biomedical engineering at the Engineering Faculty of Pisa. Since 1992, he teaches regular courses on biomedical signal and image processing at Electronic Engineering degree of the University of Pisa. He is currently engaged in research on biomedical engineering at the Information Department, Faculty of Engineering of Pisa University. He has published about 200 reports and papers on ultrasonic tissue characterization, digital signal and image processing, and medical imaging. 